\title{
Oxalidaceae endémicas del Perú
}

\author{
Blanca León ${ }^{1,2}$, Christhian Monsalve ${ }^{1}$ y Eve Emschweiler ${ }^{3}$
}

${ }^{1}$ Museo de Historia Natural, Av. Arenales 1256, Aptdo. 14-0434, Lima 14, Perú.

christhianml@hotmail.com

2 Plant Resources Center, University of Texas at Austin, Austin TX 78712 EE.UU.

blanca.leon@mail.utexas.edu ${ }^{3}$ Department of Botany, The Field Museum, 1400 S Lake Shore Drive, Chicago, IL 60605, EE.UU.

emshwill@fieldmuseum.org

\section{Resumen}

Esta es la familia de la «oca» y es reconocida en el Perú por presentar cuatro géneros y 105 especies (Brako \& Zarucchi, 1993; Ulloa Ulloa et al., 2004), principalmente arbustos y hierbas. En este trabajo reconocemos en tres géneros, 20 especies y siete taxones subespecíficos como endemismos peruanos. Los taxones endémicos se encuentran principalmente en las regiones Bosques Muy Húmedos Montanos, Bosques Muy Húmedos Premontanos y Mesoandina, entre los 700 y $3600 \mathrm{~m}$ de altitud. Doce taxones endémicos se encuentran representados dentro del Sistema Nacional de Áreas Naturales Protegidas por el Estado.

Palabras claves: Oxalidaceae, Perú, endemismo, plantas endémicas.

\section{Abstract}

This is the «oca» family and is represented in Peru by four genera and 105 species (Brako \& Zarucchi, 1993; Ulloa Ulloa et al., 2004), mainly shrubs and herbs. Here we recognize as endemics 20 species and seven infra-specific taxa in three genera. These endemic taxa are found mainly in Very Humid Montane Forests, Very Humid Premontane Forests and Mesoandean regions, between 700 and 3600 m elevation. Twelve endemic taxa have been recorded in the Peruvian System of Protected Natural Areas.

Keywords: Oxalidaceae, Peru, endemism, endemic plants.

\section{Biophytum amazonicum R. Knuth}

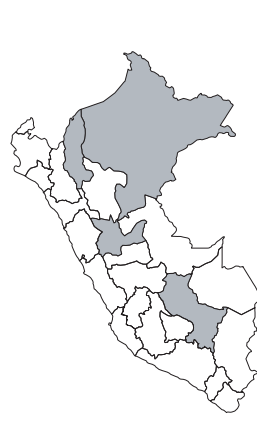

\section{NT}

Publicación: Pflanzenr. IV, 130 (Heft 95): 416. 1930.

Colección tipo: G. Tessmann s.n.

Herbarios: B.

Nombre común: Desconocido.

Registro departamental: $\mathrm{AM}, \mathrm{CU}, \mathrm{HU}$, LO.

Regiones Ecológicas: BMHP, BHA; 460-1100 m.

SINANPE: Sin registro.

Herbarios peruanos: USM (5).

Observaciones: Hierba subacaulescente conocida de varias localidades dispersas en la Amazonía peruana. Ha sido recolectada recientemente en la Cordillera del Cóndor y probablemente se encuentre en Ecuador.

\section{Biophytum foxii Sprague}

\section{DD}

Publicación: Kew Bull. 343. 1911.

Colección tipo: W. Fox s.n.

Herbarios: K.

Nombre común: Desconocido.

Registro departamental: Sin datos.

Regiones Ecológicas: Sin datos; altitud desconocida.

SINANPE: Sin registro.

Herbarios peruanos: Ninguno.

Observaciones: Herba perenne conocida de una sola localidad no determinada. Aparentemente la colección original se encuentra depositada en el herbario de Kew. No se conoce de recolectas recientes.

\section{Biophytum tessmannii R. Knuth}

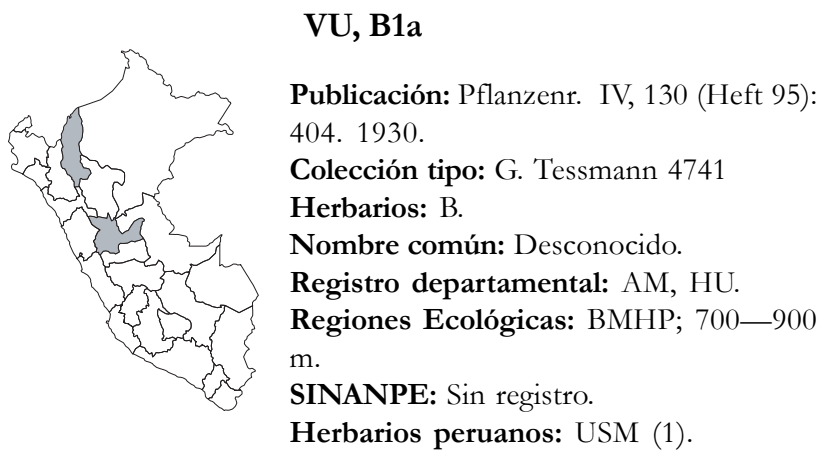

Observaciones: Hierba perenne conocida de dos localidades aisladas, en las cuencas del Huallaga y Marañón. Fue originalmente recolectada del Pongo de Manseriche en Amazonas, pero erroneamente citada como Loreto. Aparentemente, la colección más reciente proviene de 1976. Habita sotobosques no inundados, podría tratarse de una especialista de rocas calcáreas.

\section{Hypseocharis pilgeri R. Knuth}

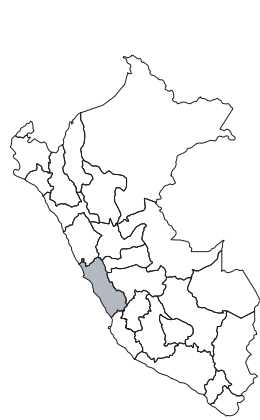

\section{EN, B1ab(iii)}

Publicación: Bot. Jahrb. Syst. 41: 174. 1908.

Colección tipo: A. Weberbauer 95

Herbarios: B.

Nombre común: Desconocido.

Registro departamental: LI.

Regiones Ecológicas: MDE, MA; 2300$2600 \mathrm{~m}$.

SINANPE: Sin registro.

Herbarios peruanos: USM (1).

Observaciones: Hierba acaulescente, conocida de la cuenca media del Rímac, donde aparentemente no ha vuelto a ser recolectada desde 1953. Crece en ambientes semixéricos dependientes de las lluvias estacionales. 


\section{Oxalis apurimacensis Lourteig}

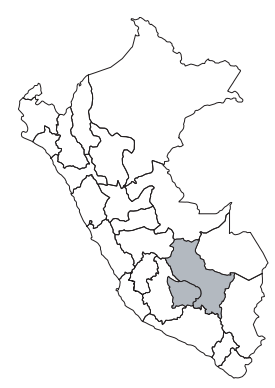

\section{DD}

Publicación: Bradea 7(2): 280, f. 70 B. 2000.

Colección tipo: C. Vargas C. 12388

Herbarios: P; $\underline{\mathrm{CUZ}}$.

Nombre común: Desconocido.

Registro departamental: AP, CU.

Regiones Ecológicas: MA; 2400—3500 m. SINANPE: SNA

Herbarios peruanos: CUZ (isotipo citado+2), USM (1).

Observaciones: Hierba perenne conocida de pocas localidades en las cuencas del Apurímac y Urubamba. Podría ser coespecífica con otra endémica, Oxalis picchensis.

\section{Oxalis bulbocastanum Phil. subsp. hirta (R. Knuth)}

\section{Lourteig}

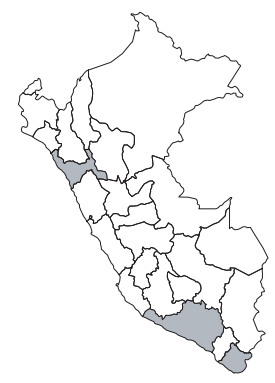

VU, B1ab(iii)

Publicación: Bradea 7(2): 356. 2000.

Colección tipo: I.M. Johnston 6281

Herbarios: GH.

Nombre común: Desconocido.

Registro departamental: AR, LL, TA.

Regiones Ecológicas: DST; $100-300 \mathrm{~m}$.

SINANPE: Sin registro.

Herbarios peruanos: CUZ (2)?, USM (5).

Observaciones: Hierba conocida de la franja costera desértica, con poblaciones naturalmente fragmentadas. Por crecer en ambientes desérticos está sujeta a cambios climáticos y afectada por la expansión agrícola y urbana. Aparentemente no ha vuelto a ser recolectada desde 1955.

\section{Oxalis carminea R. Knuth}

\section{DD}

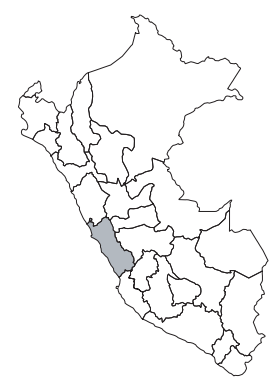

Publicación: Notizbl. Bot. Gart. BerlinDahlem 7(67): 312. 1919.

Colección tipo: A. Weberbauer 5202

Herbarios: B (d).

Nombre común: Desconocido.

Registro departamental: LI.

Regiones Ecológicas: MA; 2200—2300 m.

SINANPE: Sin registro.

Herbarios peruanos: Ninguno.

Observaciones: Hierba conocida solamente de una localidad en la cuenca media del valle del Rímac. Aparentemente no ha vuelto a ser recolectada desde 1909. Lourteig (2000) la consideró con duda en Oxalis pinguiculacea, una especie conocida del sur del Perú al norte de Argentina.

\section{Oxalis conventionensis Lourteig}

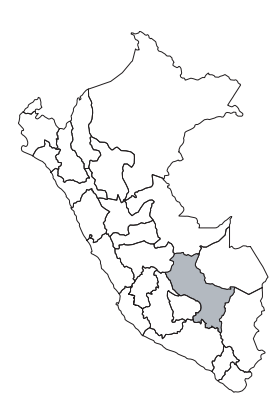

\section{DD}

Publicación: Bradea 7(2): 281. 2000.

Colección tipo: T.R. Dudley 10809

Herbarios: NA, P.

Nombre común: Desconocido.

Registro departamental: CU.

Regiones Ecológicas: BMHM; $2560 \mathrm{~m}$.

SINANPE: Sin registro.

Herbarios peruanos: Ninguno.
Observaciones: Hierba conocida, al parecer, de colecciones realizadas en 1968, en la Cordillera de Vilcabamaba, al sur del Parque Nacional Otishi. No se conoce detalles del hábitat y de la abundancia local de esta especie, pero se asume que al igual que otras en el género se presente en ambientes ecotonales.

\section{Oxalis distincta R. Knuth}

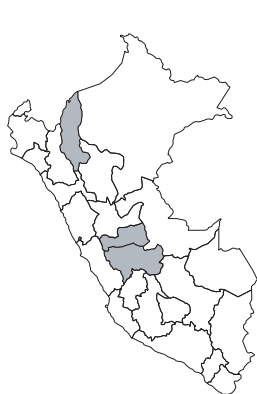

\section{VU, B1a}

Publicación: Repert. Spec. Nov. Regni Veg. 40: 292. 1936.

Colección tipo: E.P. Killip \& A.C. Smith 15586

Herbarios: NY, US.

Nombre común: Desconocido.

Registro departamental: AM, JU, PA.

Regiones Ecológicas: BMHM, BMHP; $1350-2700 \mathrm{~m}$.

SINANPE: PNYC

Herbarios peruanos: USM (3).

Observaciones: Hierba erecta, conocida del centro del país de cuatro localidades, una de ellas en el Parque Nacional YanachagaChemillén. Crece tanto en ambientes modificados, por procesos naturales, como los alterados por la intervención humana.

\section{Oxalis dudleii Lourteig}

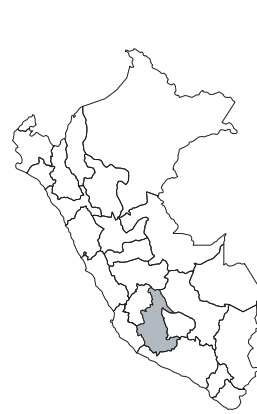

\section{DD}

Publicación: Bradea 7(2): 326, f. 81 e. 2000.

Colección tipo: T.R. Dudley 11930

Herbarios: NA, P.

Nombre común: Desconocido.

Registro departamental: AY.

Regiones Ecológicas: Sin datos; altitud desconocida.

SINANPE: Sin registro.

Herbarios peruanos: Ninguno.

Observaciones: Hierba conocida de una localidad, en la cuenca del río Apurímac. Aparentemente no ha vuelto a ser recolectada desde 1970.

\section{Oxalis humbertii Lourteig}

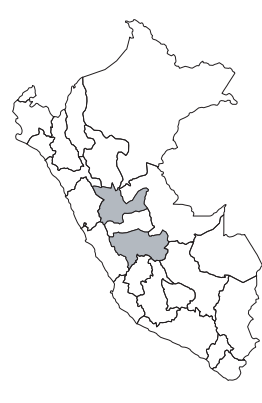

\section{VU, B1ab(iii}

Publicación: Bradea 7(2): 278, f. 69 A. 2000.

Colección tipo: A. Lourteig 3149

Herbarios: COL, K, NY, P, S, SI, US; USM.

Nombre común: Desconocido.

Registro departamental: HU, JU.

Regiones Ecológicas: BMHM; 2700$3200 \mathrm{~m}$.

SINANPE: Sin registro.

Herbarios peruanos: USM (isotipo+2).

Observaciones: Hierba del sotobosque, conocida solamente del centro del país, de las cuencas del Huallaga, Mantaro y del Monzón. Habita ambientes modificados en bosques sujetos a deforestación, por actividad humana. 


\section{Oxalis libertatis Lourteig}

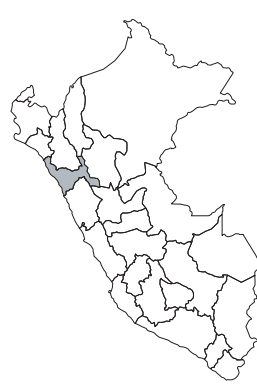
2000.

Publicación: Bradea 7(2): 215-216.

Colección tipo: S. Leiva 787

Herbarios: F, P; $\underline{\mathrm{HAO}}$.

Nombre común: Desconocido.

Registro departamental: LL.

Regiones Ecológicas: MA; $3050 \mathrm{~m}$.

SINANPE: Sin registro.

Herbarios peruanos: HAO (isotipo).

Observaciones: Esta especie trepadora se conoce de la vertiente occidental del norte del país.

\section{Oxalis lomana Diels}

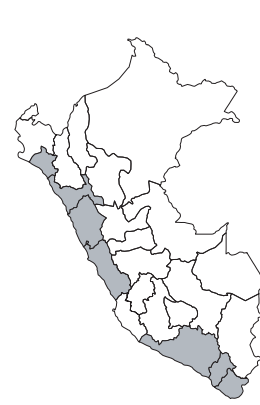

\section{NT}

Publicación: Bot. Jahrb. Syst. 37: 426. 1906. Colección tipo: C.R. Worth \& J.L. Morrison 15756

Herbarios: GH, K, MO, NA, UC.

Nombre común: Desconocido.

Registro departamental: AN, AR, LL, LA, LI, MO, TA.

Regiones Ecológicas: DST; 175-600 m. SINANPE: RNL

Herbarios peruanos: HUT (1), USM (6).

Observaciones: Hierba anual conocida de varias localidades naturalmente fragmentadas, en la costa peruana, formando parte de las comunidades de plantas de las lomas. Una subpoblación se halla en la isla San Lorenzo, frente a Lima.

\section{Oxalis lucumayensis R. Knuth subsp. lucumayensis}

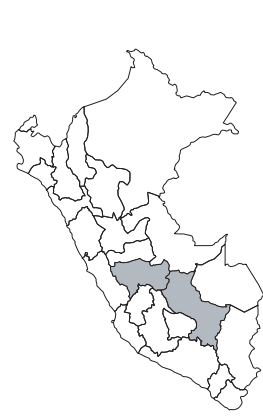

$$
\text { VU, B1a }
$$

Publicación:

Colección tipo: O.F. Cook \& G.B. Gilbert 1351

Herbarios: US.

Nombre común: Desconocido.

Registro departamental: CU, JU.

Regiones Ecológicas: BMHM; 1700$3600 \mathrm{~m}$.

SINANPE: PNM

Herbarios peruanos: USM (1).

Observaciones: Hierba postrada a trepadora, conocida de dos localidades en centro y sur del país. Aparentemente la colección más reciente data de 1983. Probablemente la extensión de su rango geográfico sea mayor, pero la escasez de registros está asociada a la taxonomía difícil del género.

\section{Oxalis lucumayensis Lourteig subsp. woytkowskii} Lourteig

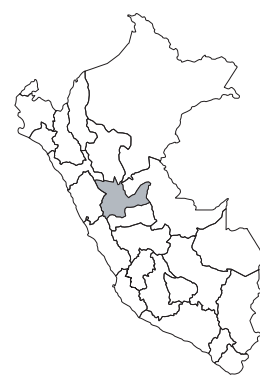

DD

Publicación: Bradea 7(2): 274, f. 68 A. 2000.

Colección tipo: F. Woytkowski 34293a

Herbarios: G, MO, P, UC.

Nombre común: Desconocido.

Registro departamental: HU.

Regiones Ecológicas: Sin datos; $3100 \mathrm{~m}$.

SINANPE: Sin registro.

Herbarios peruanos: USM (1).

Observaciones: Este taxón herbáceo se conoce de una localidad, en el centro del Perú. Poco se sabe del hábitat de esta especie, probablemente la localidad corresponda a la región Mesoandina.

\section{Oxalis ortgiesii Regel subsp. tingoensis Lourteig}

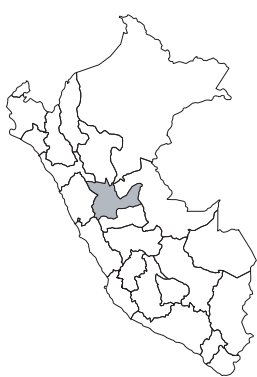

\section{EN, B1ab(iii)}

Publicación: Bradea 7(2): 326. 2000.

Colección tipo: R. Ferreyra 17004

Herbarios: $P$.

Nombre común: Desconocido.

Registro departamental: HU.

Regiones Ecológicas: BMHP; 700—750 m.

SINANPE: PNTM

Herbarios peruanos: USM (1).

Observaciones: Este taxón herbáceo se conoce solamente de una localidad, en los alrededores de Tingo María. Aparentemente está restringida a los afloramientos de roca calcárea. La colección más reciente data de 1972.

\section{Oxalis picchensis R. Knuth}

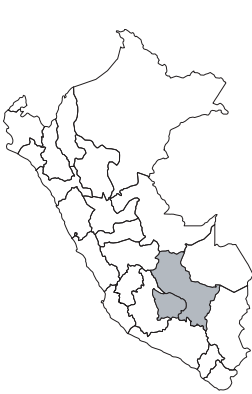

\section{VU, B1a}

Publicación: Repert. Spec. Nov. Regni Veg. 29: 214-215. 1931.

Colección tipo: F. Herrera 2806

Herbarios: B.

Nombre común: Oca oca, Oca chuchullcu. Registro departamental: AP, CU.

Regiones Ecológicas: PSH; 3200-3650 m. SINANPE: SNA

Herbarios peruanos: CUZ (1).

Observaciones: Hierba conocida de pajonales en cinco localidades del país, en las cuencas del Urubamba y Apurímac. Probablemente la extensión de su rango geográfico sea mayor, pero la escasez de registros está asociada a la taxonomía difícil del género.

\section{Oxalis pickeringii A. Gray}

\section{VU, B1a}

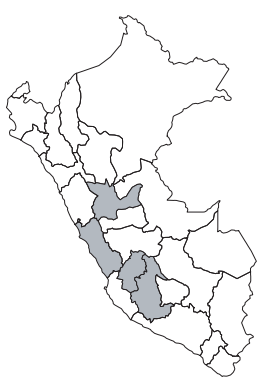

Publicación: U.S. Expl. Exped., Phan. 1: 323-324. 1854.

Colección tipo: C. Wilkes, Exped. Expl. US. s.n.

Herbarios: US.

Nombre común: Desconocido.

Registro departamental: AY, HU, HV, LI. Regiones Ecológicas: DST, MDE; 800$1500 \mathrm{~m}$.

SINANPE: Sin registro.

Herbarios peruanos: USM (1).

Observaciones: Hierba anual conocida, principalmente, de localidades en la vertiente occidental. La localidad original está ubicada en la cuenca alta del río Marañón. Al parecer, la colección de herbario más reciente proviene de 1962. La escasez de registros en los herbarios podría deberse a lo poco herborizado de las cuencas $\mathrm{y}$ ambientes en las que crece. 


\section{Oxalis ptychoclada Diels var. trichocarpa Lourteig}

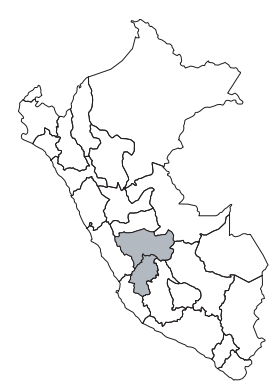

\section{EN, B1a}

Publicación: Bradea 7(2): 338. 2000. 10344

Herbarios: G, K, NA, UC.

Nombre común: Desconocido. Registro departamental: HV, JU. SINANPE: Sin registro. Colección tipo: H.E. Stork \& O.B. Horton

Regiones Ecológicas: MA; 3100—3250 m.

Herbarios peruanos: USM (3).

Observaciones: Subarbusto conocido de la cuenca media del río Mantaro. Escasamente recolectada, tal vez por el carácter decíduo de sus hojas. Ocupa ambientes en laderas con suelo rocoso, en matorrales y bosques degradados.

\section{Oxalis salticola Lourteig}

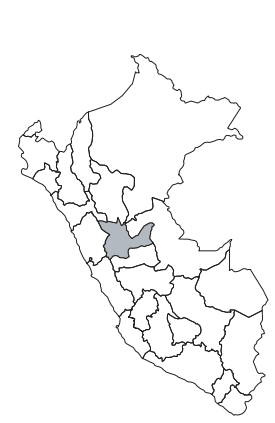

\section{EN, B1ab(iii)}

Publicación: Bradea 7(2): 314, f. 80 B. 2000.

Colección tipo: R. Ferreyra 9412

Herbarios: P; USM!.

Nombre común: Desconocido.

Registro departamental: HU.

Regiones Ecológicas: BMHM; 2250$2850 \mathrm{~m}$.

SINANPE: Sin registro.

Herbarios peruanos: USM (isotipo+5).

Observaciones: Hierba conocida de unas pocas poblaciones vecinas, en la cuenca del Huallaga. Ha sido registrada en bosques de los alrededores de Carpish, creciendo en el sotobosque como epífita o terrestre. Amenazas a sus poblaciones están asociadas a la deforestación.

\section{Oxalis san-miguelii R. Knuth subsp. san-miguelii}

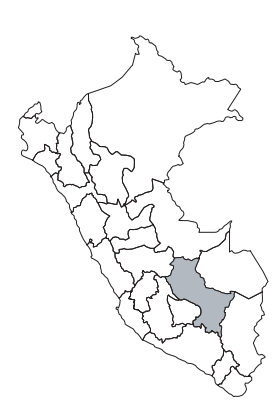

\section{EN, B1a}

Publicación:

Colección tipo: F. Herrera 2044

Herbarios: F, G, K.

Nombre común: Desconocido.

Registro departamental: CU.

Regiones Ecológicas: BMHM; 2400$2800 \mathrm{~m}$.

SINANPE: SHMP

Herbarios peruanos: USM (3).

Observaciones: Hierba perenne conocida del valle del Urubamba, donde habita tanto ambientes abiertos como boscosos. Aparentemente, no ha sido recolectada de fuera del Santuario Histórico Machu Picchu. La escasez de las recolectas botánicas podría deberse a las dificultades taxonómicas del género.

22. Oxalis san-miguelii R. Knuth subsp. urubambensis (R. Knuth) Lourteig

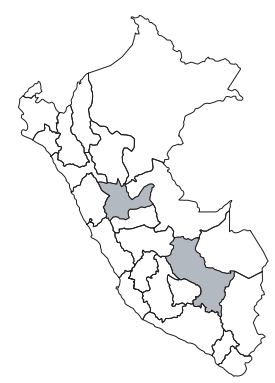

\section{EN, B1a}

Publicación: Bradea 7(2): 340. 2000.

Colección tipo: Tutin 1347

Herbarios: BM.

Nombre común: Desconocido.

Registro departamental: CU, HU.

Regiones Ecológicas: MA, BMHM; 2000-2500 m.

SINANPE: SHMP

Herbarios peruanos: CUZ (1), HUT (1), USM (1).
Observaciones: Subarbusto pequeño, conocido de ambientes ecotonales bosque- matorral. Ha sido registrada en dos localidades, una en la cuenca del Urubamba y la otra en la del Huallaga. Al parecer, no ha vuelto a ser registrada desde 1965.

\section{Oxalis semitruncata Lourteig}

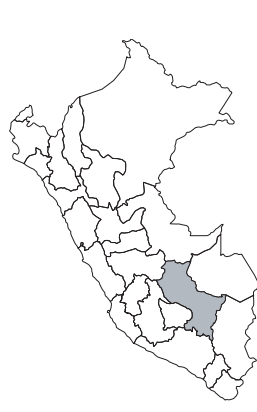

\section{EN, B1a}

Publicación: Bradea 7(2): 322, f. 81 c. 2000.

Colección tipo: M.T. Madison 10115

Herbarios: NA, P.

Nombre común: Desconocido.

Registro departamental: CU.

Regiones Ecológicas: BMHM; 1650$1757 \mathrm{~m}$.

SINANPE: PNO

Herbarios peruanos: USM (2).

Observaciones: Bejuco conocido de localidades vecinas, en la cuenca del Apurímac. Al parecer, su rango altitudinal es pequeño, el cual no supera los $200 \mathrm{~m}$. Fue descrita de una planta recolectada en la Cordillera Vilcabamba en 1970 y vuelta a registrar en 1998. La escasez de las colecciones botánicas refleja de alguna manera las dificultades taxonómicas.

\section{Oxalis vargasii Lourteig}

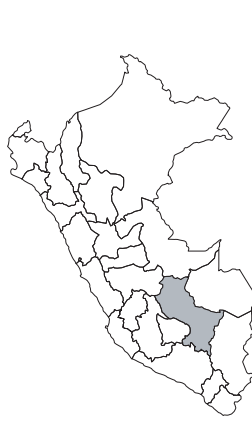

VU, B1a

Publicación: Bradea 7(2): 271-272. 2000.

Colección tipo: C. Vargas C. 2759

Herbarios: MO; $\underline{\mathrm{CUZ}}$.

Nombre común: Desconocido.

Registro departamental: CU.

Regiones Ecológicas: BPM, BMHM; 2260-3500 m.

SINANPE: PNM

Herbarios peruanos: CUZ (holotipo citado), USM (2).

Observaciones: Hierba conocida de escasas poblaciones, en las cuencas del Urubamba y Alto Madre de Dios. Aparentemente no ha vuelto a ser recolectada desde 1978. Al igual que otros taxones, la escasez de las colecciones botánicas refleja de alguna manera las dificultades taxonómicas.

\section{Oxalis westii Lourteig}

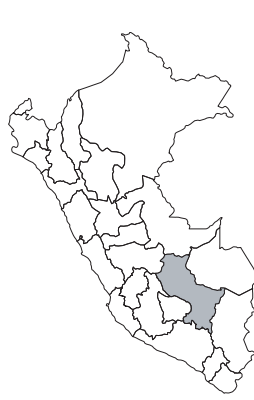

VU, B1a

Publicación: Bradea 7(2): 260, f. 65. 2000. Colección tipo: J. West 7054

Herbarios: GH, MO, US.

Nombre común: Desconocido.

Registro departamental: CU.

Regiones Ecológicas: BPM, BMHM; 2500-3500 m.

SINANPE: PNM

Herbarios peruanos: USM (4).

Observaciones: Hierba postrada, reconocible por sus tallos rojizos, conocida de varias localidades, en los alrededores del límite suroccidental del Parque Nacional Manu. Ocupa tanto ambientes boscosos como abiertos entre pajonales. Probablemente se encuentre en otras localidades a lo largo de la cordillera. 


\section{Oxalis williamsii R. Knuth}

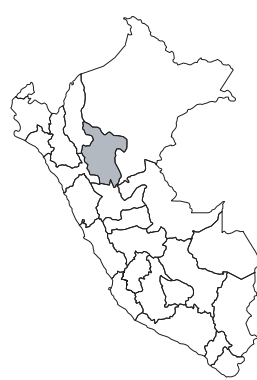

\section{DD}

Publicación: Repert. Spec. Nov. Regni Veg. 38: 195. 1935.

Colección tipo: L. Williams 7751

Herbarios: F.

Nombre común: Desconocido. Registro departamental: SM.

Regiones Ecológicas: BMHM; $1400 \mathrm{~m}$.

SINANPE: Sin registro.

Herbarios peruanos: Ninguno.

Observaciones: Arbusto conocido de una localidad de un bosque montano en San Martín. Probablemente coespecífica con Oxalis tessmannii (Lourteig, 1994).

\section{Oxalis wurdackii Lourteig}

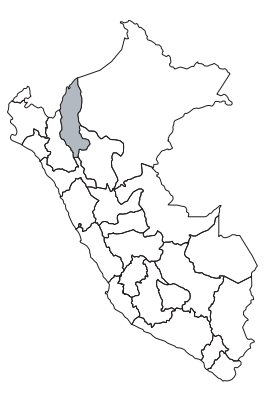

EN, B1a

Publicación: Bradea 7(2): 250-251. 2000.

Colección tipo: J.J. Wurdack 1739

Herbarios: F, G, GH, NY, P, S, US; USM.

Nombre común: Desconocido.

Registro departamental: AM.

Regiones Ecológicas: BMHM; 2900$3320 \mathrm{~m}$.

SINANPE: ZRCC

Herbarios peruanos: USM (holotipo).

Observaciones: Arbusto conocido de dos localidades, aisladas entre ellas, en el norte del país. Esta especie fue reconocida de poblaciones localmente abundantes, ocupando áreas abiertas, en Cerros Calla Calla. Conocida también de la Zona Reservada Cordillera de Colán. 